\title{
Preterminal Gasping During Hypoxic Cardiac Arrest Increases Cardiac Function in Immature Rats
}

\author{
MIOARA D. MANOLE, ROBERT W. HICKEY, NOBUO MOMOI, KIMIMASA TOBITA, JOSEPH P. TINNEY, GABRIEL P. SUCIU, \\ MICHAEL J. JOHNNIDES, ROBERT S.B. CLARK, AND BRADLEY B. KELLER
Departments of Pediatrics [M.D.M., R.W.H., NM, K.T., J.P.T., M.J.J., R.S.B.C., B.B.K.] and Critical Care Medicine [R.S.B.C.], Children's Hospital of Pittsburgh, Pittsburgh, PA 15213; Public Health Program [G.P.S.], College of Osteopathic Medicine, Nova Southeastern University, Fort Lauderdale, FL 33328

\begin{abstract}
Newborn animals are more resistant to anoxia than older animals, partly due to an increased tolerance of the immature heart to anoxia. Newborn animals also have a more robust preterminal gasp. We investigated the relationship between gasping and cardiac function in immature and maturing rats exposed to anoxia. Immature postnatal day 7 (PND7) rats $(n=13)$ and maturing PND17 rats $(n=13)$ were exposed to $100 \%$ nitrogen (anoxia) for $10 \mathrm{~min}$. Echocardiography was used to calculate cardiac contractility (CC) by left ventricular shortening fraction and cardiac output $(\mathrm{CO})$ from Doppler velocity recordings of pulmonary artery blood flow. In a separate group of PND7 rats, CC and CO were recorded after the paralytic agent pancuronium was used to prevent gasping. Anoxia decreased CC and CO in PND7 and PND17 rats, followed by a partial and transient recovery. Gasping preceded recovery of $\mathrm{CO}$ and was required to sustain CO. Gasping in PND7 rats lasted longer (541 $\mathrm{s}$ versus $351 \mathrm{~s}, p<0.01)$ and resulted in a greater recovery of $\mathrm{CC}$ and CO. Anoxia-induced gasping and the associated recovery of cardiac function were abolished by paralysis. Thus, anoxia-induced gasping transiently improves cardiac function, and more robust gasping in immature rats is associated with increased cardiac anoxic tolerance. (Pediatr Res 60: 174-179, 2006)
\end{abstract}

$\mathrm{N}^{\mathrm{e}}$ ewborn animals are more resistant to anoxia than older animals (1-4). For example, newborn rats will "autoresuscitate" after up to $20 \mathrm{~min}$ of anoxia, whereas mature rats require aggressive resuscitative efforts after even 8 min of anoxia (5). This phenomenon of neonatal tolerance to anoxia is well described but not fully understood (6). It is unknown whether the increased tolerance to anoxia in newborn animals is due to resilience of cardiac function, central nervous system (CNS) function, and/or the presence of fetal hemoglobin. Isolated heart preparations demonstrate relative tolerance to anoxia in newborn versus mature hearts (6-9). This suggests that the immature heart is inherently tolerant to anoxia. However, isolated heart preparations lack the integrated CNS and catecholamine input of live animals and lack both ventricularvascular and cardiorespiratory interactions. There are very

Received January 6, 2006; accepted March 29, 2006.

Correspondence: Mioara D. Manole, M.D., Children's Hospital of Pittsburgh, Department of Pediatrics, Division of Pediatric Emergency Medicine, 3705 Fifth Avenue, Pittsburgh, PA 15213-2583; e-mail: mioara.manole@chp.edu

This work was supported by NIH KO8 HD40848 (R.W.H.), the University of Pittsburgh Competitive Medical Research Fund (R.W.H), and NIH RO1 HL65219

(B.B.K.) limited data on cardiorespiratory interactions during anoxia in intact (in vivo) immature animals.

Gasping, also known as agonal respirations, is a universal mammalian response to anoxia. Anoxia-mediated gasping has been noted to be more robust in immature compared with mature mammals (10-12). Thus, gasping may also contribute to the phenomenon of autoresuscitation in immature mammals. Certainly, gasping facilitates respiratory air movement and resuscitation if oxygen becomes available during gasping. However, dynamic changes in intrathoracic pressure associated with gasping may also facilitate blood flow in the absence of oxygenation. Gasping-mediated blood flow augmentation was recently reported by Xie et al. (13) in a study of pigs with induced ventricular fibrillation.

In the present study, we examined the cardiorespiratory response to hypoxemia in a model of arrest that mimics the primary etiology of pediatric cardiac arrest (anoxia) $(14,15)$ using rats representing neuronal maturity equivalent to human newborns and infants (16). We measured cardiac function via echocardiography (ECHO) in two age groups of rats: immature PND7 and maturing PND17 rats before and during hypoxemia. We hypothesized that time to cardiac arrest is delayed in immature rats compared with maturing rats during hypoxemia and that preterminal gasping contributes to the relative preservation of cardiac function.

\section{METHODS}

The study protocol was approved by the Children's Hospital of Pittsburgh/ University of Pittsburgh Institutional Animal Care and Use Committee. Experiments were performed on 26 male newborn Sprague-Dawley rats, 13 male PND7 rats, and 13 male PND17 rats. The animals were exposed to anoxic conditions via administration of $100 \%$ nitrogen for $10 \mathrm{~min}$.

After determination of body weight, each rat was placed in an anesthesia chamber and received $3 \%$ isoflurane and room air (21\% oxygen). Oxygen concentration during the experiment was verified with an oxygen sensor. After onset of anesthesia ( $2 \mathrm{~min}$ ), the animal was positioned supine on a heated platform for echocardiography (ECHO) (THM 1000, VisualSonics, Toronto, Canada). Anesthetized rats breathed spontaneously and anesthesia

Abbreviations: CC, cardiac contractility; CO, cardiac output; CPR, cardiopulmonary resuscitation; ECHO, echocardiography; EDD, end-diastolic diameter; ESD, end-systolic diameter; FS, fractional shortening; LV, left ventricle; MPA, main pulmonary artery; PEA, pulseless electrical activity; PND, postnatal day

DOI: 10.1203/01.pdr.0000228333.11132.fa 
continued by using $2 \%$ isoflurane delivered via a nose cone during preparation for ECHO (9 min). Preparation for ECHO included positioning for imaging, shaving the left hemithorax, and electrocardiography (ECG) lead placement. The preanoxia anesthesia, positioning, and preparation times were similar for all groups (PND7 and PND17 rats). Baseline ECG and ECHO data were obtained after preparation in room air. We then exposed each rat to anoxic conditions using $100 \%$ nitrogen at $2 \mathrm{~L} / \mathrm{min}$ via nose cone for $10 \mathrm{~min}$. An oxygen sensor placed at the nose cone outflow confirmed $0 \%$ oxygen content within $30 \mathrm{~s}$ after initiation of anoxia. The isoflurane concentration was decreased to $1.5 \%$ for the first min of anoxia (during oxygen washout) and then discontinued for the duration of anoxia. Previous experiments using a similar anesthetic regimen have documented electroencephalography begins to recover at $2 \mathrm{~min}$ after cessation of isoflurane (17). Thus, this regimen limits the confounding effect of inhaled anesthetics while providing anesthesia during the initial stress of asphyxia.

We measured cardiac function using two complementary methods: CC measured from M-mode echocardiographic images of the beating left ventricle (LV) and $\mathrm{CO}$ measured from echocardiographic pulmonary artery blood velocity tracings. It is technically difficult to simultaneously measure both parameters on the same rat within the duration of anoxia. Therefore, rats were randomly assigned for either $\mathrm{CC}$ or $\mathrm{CO}$ measurement. We recorded ECG and ECHO data every $10 \mathrm{~s}$ throughout the experiment. We recorded ECHO for CC on six PND7 and seven PND17 rats and CO in seven PND7 and six PND17 rats. The time course of gasping onset and cessation was recorded for each rat.

ECHO. Transthoracic ECHO was performed using an Acuson Sequoia C256 system and 13-MHz linear ultrasonic transducer (15L8; Acuson Corporation, Mountain View, CA) by a skilled pediatric cardiologist. This configuration generates $0.35-\mathrm{mm}$ lateral resolution and $0.25-\mathrm{mm}$ axial resolution and is capable of acquiring and storing real-time digital images simultaneously. For CC measurement, we recorded LV short axis M-mode data at the level of the papillary muscle tips (18) and then calculated LV end-diastolic diameter (EDD), LV end-systolic diameter (ESD), and LV fractional shortening (FS) as FS $=(\mathrm{EDD}-\mathrm{ESD}) / \mathrm{EDD}$. For CO measurement, we recorded RV short axis (basal) images to measure main pulmonary artery (MPA) pulsed-Doppler velocity waveforms and the determine MPA diameter (d). We then measured the MPA velocity time integral (VTI) from individual Doppler waveforms. All Doppler recordings were obtained at an insonation angle $<15$ degrees to flow direction. Angle correction was not used. We calculated heart rate (HR) from the sequential R-R intervals on ECG waveforms, and the $\mathrm{CO}$ using the formula $\mathrm{CO}=\mathrm{HR} \cdot \mathrm{VTI} \cdot \pi \cdot \mathrm{d}^{2} / 4$. Time course changes (or temporal changes) in $\mathrm{CO}$ during anoxic exposure was expressed normalized by baseline $\mathrm{CO}$ for each rat before anoxia [\% change $\mathrm{CO}=($ anoxic $\mathrm{CO} /$ baseline $\mathrm{CO}) \times 100 \%]$.

To determine whether gasping was coincident to changes in cardiac function or causal, an additional nine PND7 rats were paralyzed with pancuronium (1.5-2 mg/kg intralingually) immediately before the onset of anoxia. Baseline and anoxia ECG and ECHO data were recorded as described above to calculate $\mathrm{CC}(n=4), \mathrm{CO}(n=5)$, and gasping pattern. We chose PND7 rats for paralysis due to their more robust gasp response to anoxia versus PND17 rats.

Statistics. Baseline measurements were compared using the nonparametric Wilcoxon rank-sum test because normality assumptions were violated. In the time-to-event analysis, we used the Kaplan-Meier method and the log-rank test because the proportional hazards assumption was satisfied (19). The trend patterns in the graphical representation of the scatter plots were illustrated using smoothing spline fits. Data were presented for baseline evaluation as means $\pm \mathrm{SD}$. We used two-sided hypotheses testing with a type I error of 0.05 . A $p$ value $<0.05$ was considered statistically significant. The analysis was performed using the SAS statistical package (version 9.1) and SAS-JMP (version 5.1).

\section{RESULTS}

Baseline values for weight, $\mathrm{HR}, \mathrm{CO}$, and $\mathrm{CC}$ are summarized in Table 1. As expected, weight and baseline HR differed between the two groups (PND7 versus PND17 rats, $p<0.05$ for each measure). Baseline $\mathrm{CC}$ differed between the two groups (PND7 versus PND17 rats, $p<0.05$ ); however, baseline $\mathrm{CO}$ was similar.

Time to cardiac arrest during anoxia. Cardiac arrest was defined by the absence of measurable $\mathrm{CC}$ or $\mathrm{CO}$ sustained for 1 min. Mean time to anoxia-induced cessation of $\mathrm{CC}$ was
Table 1. Baseline values for immature PND7 and maturing PND17 rats

\begin{tabular}{lccc}
\hline & $\begin{array}{c}\text { PND7 rats } \\
(n=13\end{array}$ & $\begin{array}{c}\text { PND17 rats } \\
(n=13)\end{array}$ & $p^{*}$ \\
\hline Weight $(\mathrm{g})$ & $17.7 \pm 2.1$ & $30.0 \pm 1.3$ & $<0.001$ \\
HR baseline $(\mathrm{bpm})$ & $336 \pm 22$ & $376 \pm 38$ & 0.0037 \\
CO baseline $(\mathrm{mL} / \mathrm{min})$ & $23.7 \pm 9.8$ & $33.5 \pm 5.4$ & 0.0741 \\
CC baseline & $0.29 \pm 0.02$ & $0.34 \pm 0.04$ & 0.0266 \\
\hline
\end{tabular}

$* p$ Values are based on the two-sided Wilcoxon rank-sum test.

Data are presented as mean $\pm \mathrm{SD}$. bpm, beats per minute.

$541 \mathrm{~s}(\mathrm{SD}=48 \mathrm{~s})$ in the PND7 group and $351 \mathrm{~s}(\mathrm{SD}=54 \mathrm{~s})$ in the PND17 group (Fig. 1, log-rank test, $p<0.01$ ). Measurable $\mathrm{CO}$ was maintained longer than $600 \mathrm{~s}$ in PND7 rats versus a maximum of $450 \mathrm{~s}$ in PND17 rats (Fig. 2, log-rank test, $p<0.01)$. In contrast to $\mathrm{CC}$, measurable $\mathrm{CO}$ persisted throughout hypoxemia in PND7 rats. There was a similar decline in HR in response to hypoxemia for both groups (Fig. 3).

Association of anoxia induced gasping and CC. After an initial decrease in $\mathrm{CC}$ in response to hypoxemia, both groups of rats showed a transient partial CC recovery coincident with gasping (Figs. 4 and 5). Mean maximal CC recovery to $100 \%$ of baseline occurred in PND7 rats versus recovery to 64\% of baseline in PND17 rats. This percentage of recovery represents the mean of maximum values of $\mathrm{CC}$ attained by each animal after 1 min of anoxia. Representative ECHO images demonstrating recovery of $\mathrm{CC}$ during gasping in a PND7 rat are shown in Figure 5. The time course of the $\mathrm{CC}$ response to gasping differed between PND7 and PND17 rats $(p<0.0001)$. Onset of gasping was delayed in PND7 rats but persisted for a longer duration compared with PND17 rats (onset $324 \mathrm{~s}$ and duration $165 \mathrm{~s}$ versus onset $157 \mathrm{~s}$ and duration $80 \mathrm{~s}$, respectively). Once gasping ceased (489 s for PND7 and 237 s for PND17), CC decreased toward zero. PND7 rats paralyzed with pancuronium to ablate gasping showed a progressive decline of $\mathrm{CC}$ during anoxic conditions without recovery (Fig. 6).

Association of anoxia-induced gasping and CO. After an initial decrease in $\mathrm{CO}$ during hypoxemia, both groups of rats showed a transient partial CO recovery coincident with gasping (Figs. 7 and 8). Mean maximal CO recovery to $40 \%$ of

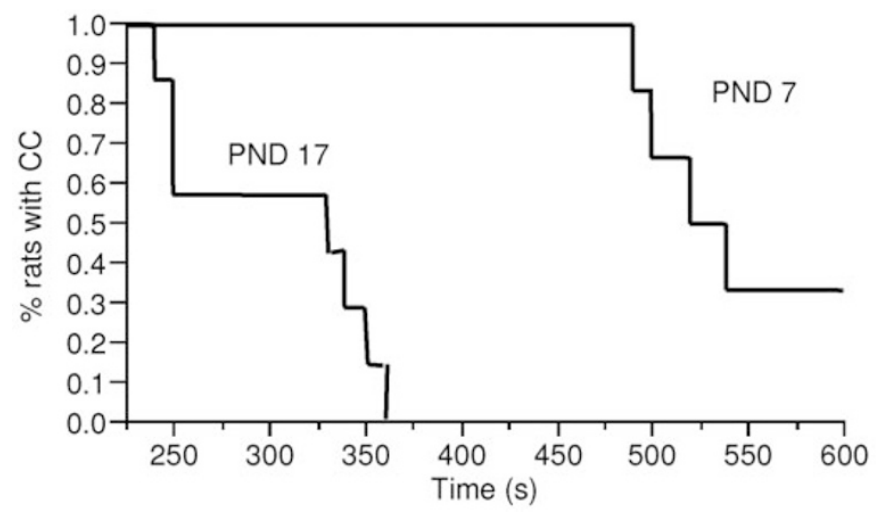

Figure 1. Survival plot demonstrating the time to cardiac arrest defined as cessation of CC after the onset of anoxia in PND7 and PND17 rats (13 rats in each group, log-rank test, $p<0.01$ ). 


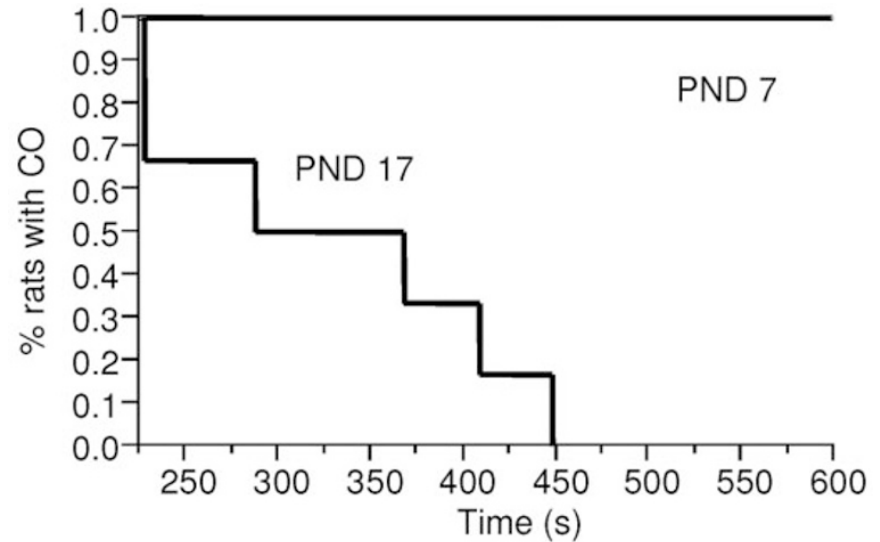

Figure 2. Survival plot demonstrating the time to cardiac arrest defined as cessation of $\mathrm{CO}$ after the onset of anoxia in PND7 and PND17 (13 in each group, logrank test, $p<0.01$ ).
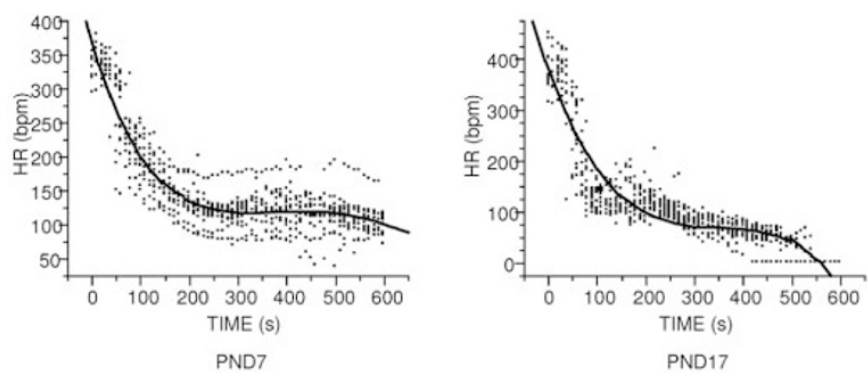

Figure 3. Pattern of HR decrease during anoxia in PND7 and PND17 rats. Individual data points from each rat and a curve depicting the mean values are displayed. HR decline was similar in time course and extent between the two groups.

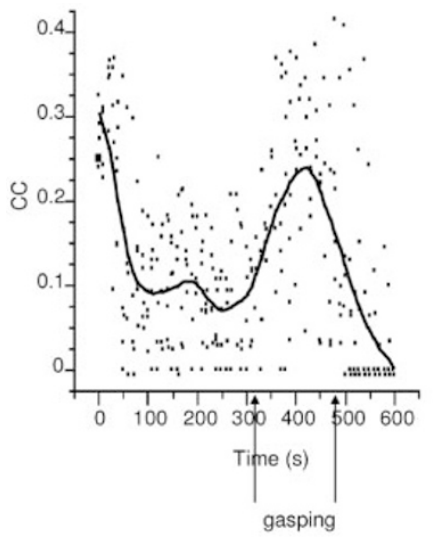

PND 7

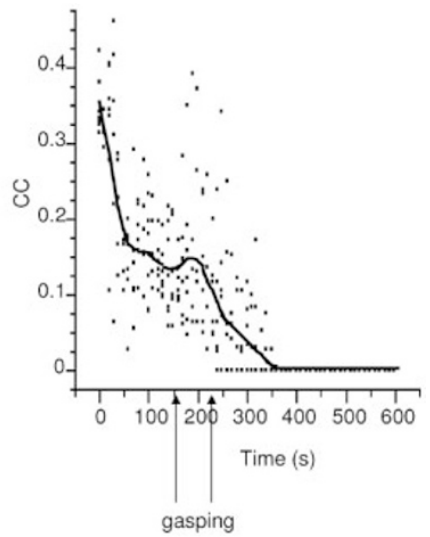

PND 17
Figure 4. Time course of CC during anoxia in PND7 (left) and PND17 (right) rats. Individual data points from each rat and a curve depicting the mean values are displayed. The onset and end of gasping are noted by the black arrows. Note the increase in CC that occurs during gasping in PND7 rats and the early absence of CC in PND17 rats.

baseline occurred in PND7 rats versus to $25 \%$ of baseline in PND17 rats. This percentage of recovery represents the mean of maximum values of $\mathrm{CO}$ attained by each animal after $1 \mathrm{~min}$ of anoxia. Representative ECHO images demonstrating recovery of $\mathrm{CO}$ during gasping in a PND7 rat are shown in Figure 8 . The time course of the $\mathrm{CO}$ response to gasping differed between PND7 and PND17 rats $(p<0.0001)$. Once gasping ceased (489 s for PND7 and $237 \mathrm{~s}$ for PND17), CO

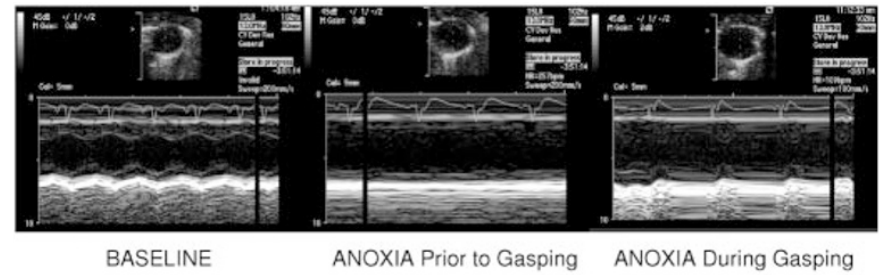

Figure 5. Representative two-dimensional and M-mode ECHO images of a PND7 rat demonstrating normal CC at baseline, marked decrease in CC during anoxia before gasping, and partial recovery of $\mathrm{CC}$ during gasping. Note the slower HR during anoxia.

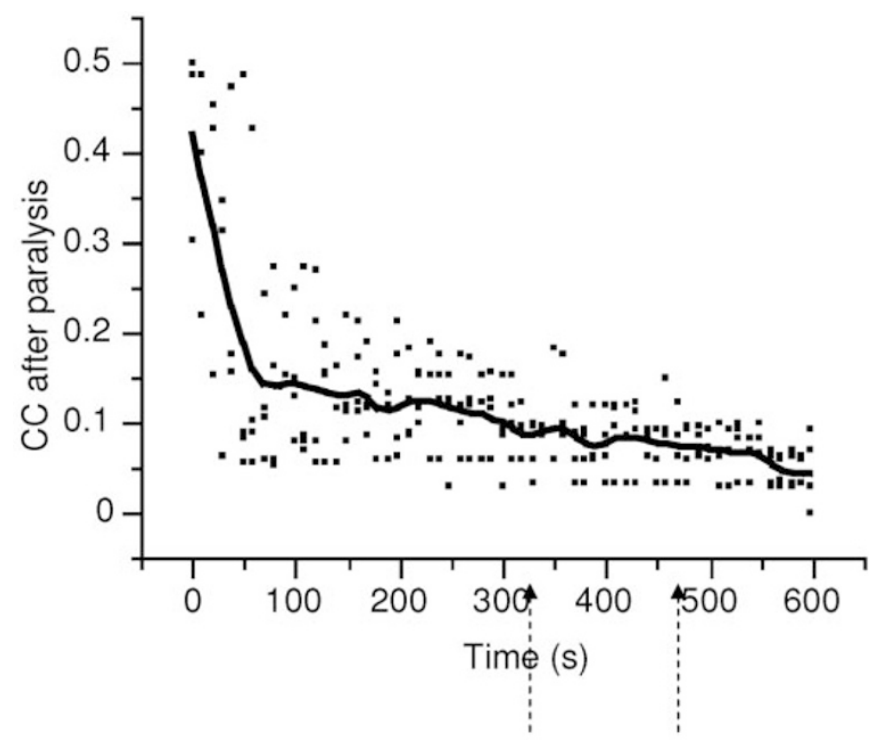

Figure 6. Time course of $\mathrm{CC}$ during anoxia in paralyzed PND7. Individual data points from each rat and a curve depicting the mean values are displayed. The time of onset and end of gasping in nonparalyzed PND7 rats are noted by the dashed arrows. Note the progressive decline in CC during anoxia in paralyzed PND7 rat and the absence of transient CC recovery.

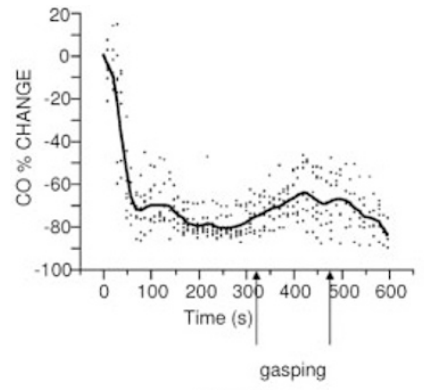

PND 7

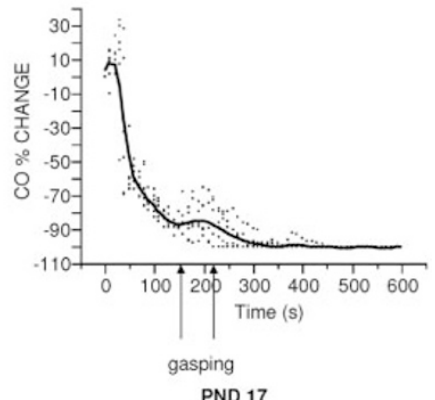

PND 17
Figure 7. Time course of $\mathrm{CO}$ measured as pulmonary blood flow during anoxia in PND7 (left) and PND17 (right) rats. Individual data points from each rat and a curve depicting the mean values are displayed. The onset and end of gasping are noted by the black arrows. Note the increase in $\mathrm{CO}$ that occurs during gasping in PND7 rats and the early absence of CO in PND17 rats.

decreased toward zero. PND7 rats paralyzed with pancuronium to ablate gasping showed a progressive decline of $\mathrm{CO}$ during anoxic conditions without recovery (Fig. 9).

\section{DISCUSSION}

To our knowledge, this is the first study that assesses in vivo $\mathrm{CC}$ and $\mathrm{CO}$ during anoxic conditions in spontaneously breath- 


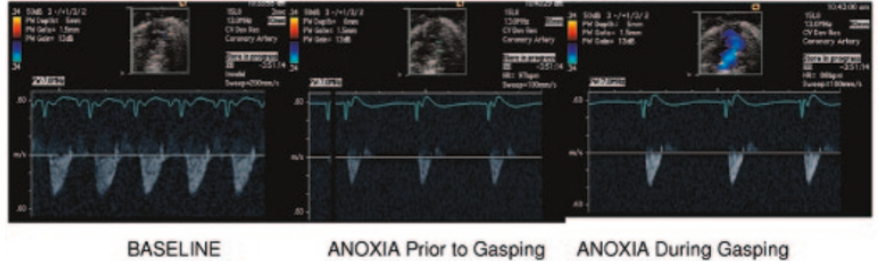

Figure 8. Representative two-dimensional ECHO and pulsed-Doppler velocity tracings of pulmonary blood flow of a PND7 rat demonstrating normal CO at baseline, marked bradycardia, and decreased $\mathrm{CO}$ during anoxia before gasping, and partial recovery of $\mathrm{CO}$ during gasping. Note the slower HR during anoxia.

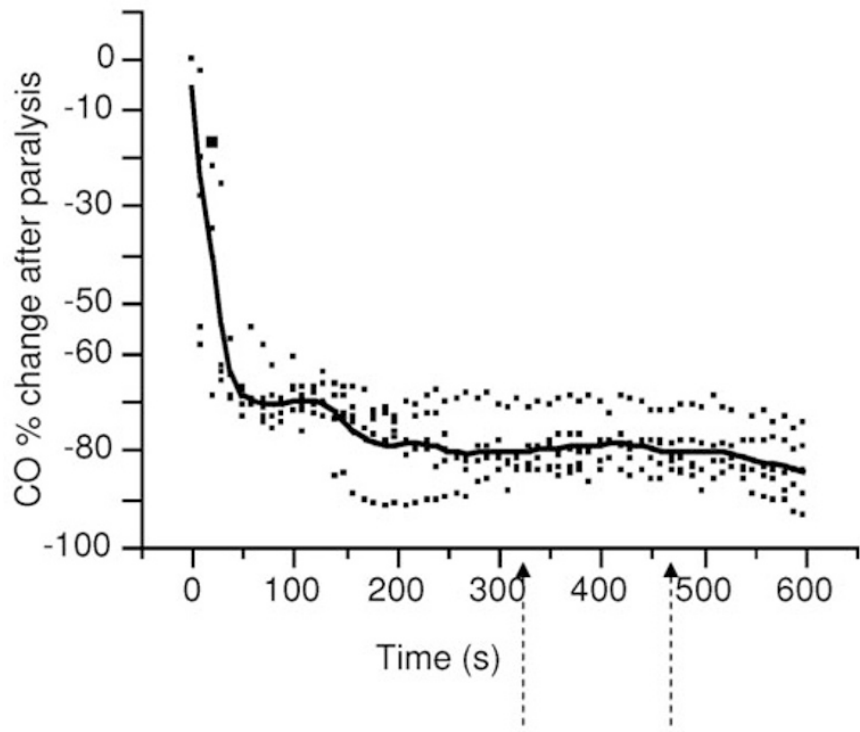

Figure 9. Time course of CO during anoxia in paralyzed PND7. Individual data points from each rat and a curve depicting the mean values are displayed. The time of onset and end of gasping in nonparalyzed PND7 rats are noted by the dashed arrows. Note the progressive decline in $\mathrm{CO}$ during anoxia in paralyzed PND7 rat and the absence of transient CO recovery.

ing immature animals. We demonstrate, using ECHO, that CC and $\mathrm{CO}$ are maintained for longer periods of time in immature animals compared with maturing animals and that gasping contributes to the maintenance of $\mathrm{CC}$ and $\mathrm{CO}$. These observations are consistent with previous studies showing that immature animals are more resistant to anoxic conditions. Isolated heart preparations have shown that hearts removed from immature animals are more resistant to anoxia than those removed from older animals related to cardiomyocyte maturation (6-9). We have extended this observation to show that tolerance to anoxia in the intact circulation occurs, in part, due to anoxia-induced gasping in immature animals. Furthermore, we show that paralysis extinguishes the beneficial effects of gasping. This suggests that gasping and cardiac recovery are not merely coincident phenomena mediated by increased stimulatory input from related CNS centers but are causally related. Preterminal gasping likely produces accentuated cyclical variations in intrathoracic pressure that facilitate forward cardiac blood flow.

Several mechanisms might be responsible for the observed increase in $\mathrm{CC}$ and $\mathrm{CO}$ during gasping. Increased $\mathrm{CC}$ might be related to an increase in $\mathrm{ILV}$ preload as the result of increased venous return in response to negative intrathoracic pressure.
Increase preload results in an increase in stroke volume due to the positive slope of the Frank-Starling curve. Another mechanism that could be responsible for this increase in $\mathrm{CC}$ is a gasping-induced increase in myocardial perfusion. Yannopolus et al. (20), using an impedance threshold device designed to increase negative intrathoracic pressure during the relaxation phase of chest compression, showed lower right atrial pressure but better coronary perfusion pressure with use of the device, and a similar mechanism could occur during gasping. Additionally, cerebral perfusion pressure increases during gasping (21). Increased cerebral perfusion pressure might help sustain sympathetic drive to the heart and promote CO. It is important to note that the magnitude of increased contractility in the current study was sustained between gasps. If improved $\mathrm{CC}$ was secondary only to improved venous return, we would expect to have observed a greater increase in contraction immediately after a gasp followed by decreased magnitude in subsequent contractions before the next gasp. With our current data, we can only speculate that the improved cardiac function is secondary to a combination of the factors mentioned above.

Our observations are consistent with the report by Xie et al. (13) that shows gasping increased $\mathrm{CO}$ in pigs during ventricular fibrillation. We chose a model of respiratory-mediated, pulseless electrical activity (PEA) arrest because respiratory compromise is the most common etiology of pediatric arrest and PEA is the most common dysrhythmia in pediatric arrest (22). PND7 rats were chosen because the cerebral maturity of a PND7 rat approximates cerebral maturity of a full-term human newborn, and PND17 rats were chosen because they approximate toddler-aged children (23).

Gasping is a common mammalian preterminal phenomenon. Agonal respirations occur in all animals during ventricular fibrillation (24). Home monitor data on infants dying of sudden infant death syndrome (SIDS) reveal that 31 of 33 infants had preterminal gasping respirations $(25,26)$. In studies of witnessed adult out-of-hospital arrest, 30-40\% of bystanders describe agonal respirations $(27,28)$. Gasping can be autoresuscitative if oxygen delivery is resumed during gasping. Previous studies show that the frequency of gasping is predictive of the success of resuscitation (29) and that gasping improves resuscitation after the onset of cardiac arrest (30). The animals in our study were kept in an anoxic environment during the gasping period, preventing autoresuscitation. Gozal et al. (11) demonstrated that there is an inverse relationship between postnatal age and gasp duration and gasp latency. Our study supports this observation, with PND7 rats having longer duration of gasping and later onset of gasping than PND17 rats.

The observation that spontaneous gasping increases $\mathrm{CO}$ has clinical relevance for techniques of performing cardiopulmonary resuscitation (CPR). Traditional CPR generates blood flow by alternating positive intrathoracic pressure (generating forward flow) and negative intrathoracic pressure (facilitating venous return to the heart). Gasping may be beneficial by causing a large negative intrathoracic pressure that increases venous return to the heart. Alternative methods of CPR have been developed that augment negative intrathoracic pressure during the relaxation phase of CPR including active compression-decompression CPR using a suction device at- 
tached to the chest (31), an impedance threshold valves on endotracheal tubes to limit passive air entry during the relaxation phase of chest compressions, thus generating greater negative intrathoracic pressure (32), and pneumatic vest resuscitation that promotes circumferential compression and expansion $(33,34)$. Our results suggest that these and other methods to augment negative intrathoracic pressure can result in potentially beneficial hemodynamic changes. Furthermore, it has recently been shown that too much positive intrathoracic pressure (caused by overinflation from incomplete exhalation) during cardiac resuscitation can be detrimental (35). Thus, mimicking the cardiopulmonary effects of gasping during $\mathrm{CPR}$ may result in improved $\mathrm{CO}$ and may ultimately be beneficial by maintaining at least partial cerebral blood flow under anoxic conditions. Additionally, researchers at the University of Minnesota have documented improved CO in response to phrenic nerve pacing induced respiratory augmentation in pigs during hemorrhagic shock (36).

Of note, children with SIDS associated with autoresuscitation failure have a reduced number of gasps (37). Experimental data suggested that gasping depends on pacemaker neurons that are relatively hypoxia resistant (38), and thus these neurons may be impaired in children with SIDS. Additional understanding of the regulation and function of the brain's gasping center may lead to pharmacologic interventions in children at risk of acute or recurrent anoxia or hypoxia.

This is the first study to use ECHO for measurement of cardiac function in immature rats. We chose to measure $\mathrm{CO}$ via VTI at the level of the MPA rather than at the level of the ascending aorta because the anatomic angle of the pulmonary artery in these rats provided a more robust signal compared with aorta. In the absence of intracardiac or intrapulmonary shunts, $\mathrm{CO}$ to the lungs and body should be identical. In pilot experiments, we compared $\mathrm{CO}$ calculated by MPA flow to $\mathrm{CO}$ calculated by LV M-mode (Pombo's cubic assumption), and we observed that the trend in $\mathrm{CO}$ during the anoxia was similar. Therefore, we concluded that estimated CO by MPA flow reflected LV CO (systemic flow).

We noted a difference in baseline $\mathrm{CO}$ and $\mathrm{CC}$ between the PND7 and PND17 groups. CO at baseline appears to differ between groups; however, the difference is not statistically significant. If we normalize $\mathrm{CO}$ by body weight, the values converge to $1.34 \mathrm{~mL} / \mathrm{min} / \mathrm{g}$ (PND7) and $1.13 \mathrm{~mL} / \mathrm{min} / \mathrm{g}$ (PND17). We noted that baseline CC differed between the two groups, with higher CC measured in PND17 hearts, possibly related to myocyte maturation (39).

We recognize that there are limitations to this study. Inhalational anesthetics, including isoflurane, can be cardioprotective (40). However, it would be unethical to initiate anoxia in the absence of anesthesia. We attempted to limit the isoflurane effect on cardiac function by discontinuing isoflurane 1 min after the onset of anoxia. Another limitation of our study is that $\mathrm{CO}$ and $\mathrm{CC}$ were measured in separate cohorts of rats instead of instantaneously in the same animals. This was necessary because pilot studies showed that moving the probe from one measurement to the other required excessive time to reestablish the probe angle and location. Similarly, measuring
$\mathrm{CO}$ via intravascular catheters would have been ideal, but difficult due to the size of the animals.

\section{CONCLUSION}

During acute anoxic conditions, $\mathrm{CC}$ and $\mathrm{CO}$ are maintained for longer periods of time in immature animals compared with maturing animals. This tolerance to anoxia is mediated, in part, by an anoxia-induced gasping, which can be ablated by muscle paralysis. Further studies are needed to define the regulation of gasping in immature and mature animals with the goal of optimizing $\mathrm{CC}$ and $\mathrm{CO}$ during hypoxemia.

Acknowledgment. The authors thank Dr. Richard A. Saladino for his valuable guidance and for reviewing the manuscript.

\section{REFERENCES}

1. Fazekas JF, Alexander FA, Himwich HE 1941 Tolerance of the newborn to anoxia. Am J Physiol 134:281-287

2. Duffy TE, Kohle SJ, Vannucci RC 1975 Carbohydrate and energy metabolism in perinatal rat brain: relation to survival in anoxia. J Neurochem 24:271-276

3. Stafford A, Weatherall JA 1960 The survival of young rats in nitrogen. J Physiol 153:457-472

4. Le Gallois C 1812 Experiences sur le principe de la vie. Chez d'Hautel, Paris, pp 78-79

5. Kobrin VI, Ignatova ED 1990 [Cardiac fibrillation in early ontogeny in animals born mature and immature]. Fiziol Zh SSSR Im I M Sechenova 76:1317-1320

6. Singer D 1999 Neonatal tolerance to hypoxia: a comparative-physiological approach. Comp Biochem Physiol A Mol Integr Physiol 123:221-234

7. Awad WI, Shattock MJ, Chambers DJ 1998 Ischemic preconditioning in immature myocardium. Circulation 98:II206-II213

8. Bove EL, Stammers AH 1986 Recovery of left ventricular function after hypothermic global ischemia. Age-related differences in the isolated working rabbit heart. J Thorac Cardiovasc Surg 91:115-122

9. Yano Y, Braimbridge MV, Hearse DJ 1987 Protection of the pediatric myocardium. Differential susceptibility to ischemic injury of the neonatal rat heart. J Thorac Cardiovasc Surg 94:887-896

10. Fewell JE, Smith FG, Ng VK, Wong VH, Wang Y 2000 Postnatal age influences the ability of rats to autoresuscitate from hypoxic-induced apnea. Am J Physiol Regul Integr Comp Physiol 279:R39-R46

11. Gozal D, Torres JE, Gozal YM, Nuckton TJ 1996 Characterization and developmental aspects of anoxia-induced gasping in the rat. Biol Neonate 70:280 288

12. Gozal D, Torres JE 1997 Maturation of anoxia-induced gasping in the rat: potential role for N-methyl-D-aspartate glutamate receptors. Pediatr Res 42:872-877

13. Xie J, Weil MH, Sun S, Yu T, Tang W 2004 Spontaneous gasping generates cardiac output during cardiac arrest. Crit Care Med 32:238-240

14. Young KD, Seidel JS 1999 Pediatric cardiopulmonary resuscitation: a collective review. Ann Emerg Med 33:195-205

15. Young KD, Gausche-Hill M, McClung CD, Lewis RJ 2004 A prospective, population-based study of the epidemiology and outcome of out-of-hospital pediatric cardiopulmonary arrest. Pediatrics 114:157-164

16. Hagberg H, Bona E, Gilland E, Puka-Sundvall M 1997 Hypoxia-ischaemia model in the 7-day-old rat: possibilities and shortcomings. Acta Paediatr Suppl 422:85-88

17. Fink EL, Alexander H, Marco CD, Dixon CE, Kochanek PM, Jenkins LW, Lai Y, Donovan HA, Hickey RW, Clark RS 2004 Experimental model of pediatric asphyxial cardiopulmonary arrest in rats. Pediatr Crit Care Med 5:139-144

18. Gardin JM, Siri FM, Kitsis RN, Edwards JG, Leinwand LA 1995 Echocardiographic assessment of left ventricular mass and systolic function in mice. Circ Res 76:907914

19. Suciu G, Lemeshow S, Moeschberger M 2004 Statistical Test of Equality of Survival Curves: Reconsidering the Options. In: Balakrishnan N, Rao CR (eds) Handbook of Statistics (vol 23). Elsevier Science, North-Holland, pp 251-262

20. Yannopoulos D, Nadkarni VM, McKnite SH, Rao A, Kruger K, Metzger A, Benditt DG, Lurie KG 2005 Intrathoracic pressure regulator during continuous-chestcompression advanced cardiac resuscitation improves vital organ perfusion pressures in a porcine model of cardiac arrest. Circulation 112:803-811

21. Srinivasan V, Nadkarni VM, Yannopoulos D, Marino BS, Sigurdsson G, McKnite SH, Zook M, Benditt DG, Lurie KG 2006 Spontaneous gasping decreases intracranial pressure and improves cerebral perfusion in a pig model of ventricular fibrillation. Resuscitation 69:329-334

22. Donoghue AJ, Nadkarni V, Berg RA, Osmond MH, Wells G, Nesbitt L, Stiell IG 2005 Out-of-hospital pediatric cardiac arrest: an epidemiologic review and assessment of current knowledge. Ann Emerg Med 46:512-522 
23. Rice D, Barone S Jr 2000 Critical periods of vulnerability for the developing nervous system: evidence from humans and animal models. Environ Health Perspect 108(suppl 3):511-533

24. Menegazzi JJ, Check BD 1995 Spontaneous agonal respiration in a swine model of out-of-hospital cardiac arrest. Acad Emerg Med 2:1053-1056

25. Poets CF, Meny RG, Chobanian MR, Bonofiglo RE 1999 Gasping and other cardiorespiratory patterns during sudden infant deaths. Pediatr Res 45:350354

26. Sridhar R, Thach BT, Kelly DH, Henslee JA 2003 Characterization of successful and failed autoresuscitation in human infants, including those dying of SIDS. Pediatr Pulmonol 36:113-122

27. Clark JJ, Larsen MP, Culley LL, Graves JR, Eisenberg MS 1992 Incidence of agonal respirations in sudden cardiac arrest. Ann Emerg Med 21:1464-1467

28. Bang A, Herlitz J, Martinell S 2003 Interaction between emergency medical dispatcher and caller in suspected out-of-hospital cardiac arrest calls with focus on agonal breathing. A review of 100 tape recordings of true cardiac arrest cases. Resuscitation 56:25-34

29. Yang L, Weil MH, Noc M, Tang W, Turner T, Gazmuri RJ 1994 Spontaneous gasping increases the ability to resuscitate during experimental cardiopulmonary resuscitation. Crit Care Med 22:879-883

30. Kloss T, Roewer N, Wischhusen F 1985 [Prognosis of preclinical cardiopulmonary resuscitation]. Anasth Intensivther Notfallmed 20:237-243

31. Plaisance P, Lurie KG, Vicaut E, Adnet F, Petit JL, Epain D, Ecollan P, Gruat R, Cavagna P, Biens J, Payen D 1999 A comparison of standard cardiopulmonary resuscitation and active compression-decompression resuscitation for out-of-hospital cardiac arrest. French Active Compression-Decompression Cardiopulmonary Resuscitation Study Group. N Engl J Med 341:569-575
32. Plaisance P, Lurie KG, Payen D 2000 Inspiratory impedance during active compression-decompression cardiopulmonary resuscitation: a randomized evaluation in patients in cardiac arrest. Circulation 101:989-994

33. Niemann JT, Rosborough JP, Niskanen RA, Criley JM 1984 Circulatory support during cardiac arrest using a pneumatic vest and abdominal binder with simultaneous high-pressure airway inflation. Ann Emerg Med 13:767-770

34. Ben Haim SA, Shofti R, Ostrow B, Dinnar U 1989 Effect of vest cardiopulmonary resuscitation rate on cardiac output and coronary blood flow. Crit Care Med $17: 768-771$

35. Aufderheide TP, Sigurdsson G, Pirrallo RG, Yannopoulos D, McKnite S, von Briesen C, Sparks CW, Conrad CJ, Provo TA, Lurie KG 2004 Hyperventilation-induced hypotension during cardiopulmonary resuscitation. Circulation 109:1960-1965

36. Samniah N, Voelckel WG, Zielinski TM, McKnite S, Patterson R, Benditt DG, Lurie KG 2003 Feasibility and effects of transcutaneous phrenic nerve stimulation combined with an inspiratory impedance threshold in a pig model of hemorrhagic shock. Crit Care Med 31:1197-1202

37. Poets CF 2004 Apparent life-threatening events and sudden infant death on a monitor. Paediatr Respir Rev 5(suppl A):S383-S386

38. Pena F, Parkis MA, Tryba AK, Ramirez JM 2004 Differential contribution of pacemaker properties to the generation of respiratory rhythms during normoxia and hypoxia. Neuron 43:105-117

39. Engelmann GL, Campbell SE, Rakusan K 1996 Immediate postnatal rat heart development modified by abdominal aortic banding: analysis of gene expression. Mol Cell Biochem 163-164:47-56

40. Stowe DF, Kevin LG 2004 Cardiac preconditioning by volatile anesthetic agents: defining role for altered mitochondrial bioenergetics. Antioxid Redox Signal 6:439448 